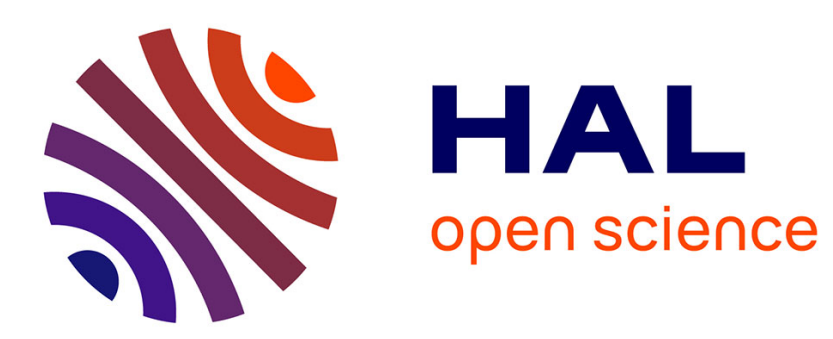

\title{
Micromechanical modelling of the elastoplastic behaviour of metallic material under strain-path changes
} Jamal Fajoui, David Gloaguen, Bruno Courant, Ronald Guillen

\section{To cite this version:}

Jamal Fajoui, David Gloaguen, Bruno Courant, Ronald Guillen. Micromechanical modelling of the elastoplastic behaviour of metallic material under strain-path changes. Computational Mechanics, 2009, 44 (2), pp.285-296. 10.1007/s00466-009-0374-7 . hal-01007041

\section{HAL Id: hal-01007041 \\ https://hal.science/hal-01007041}

Submitted on 10 Dec 2017

HAL is a multi-disciplinary open access archive for the deposit and dissemination of scientific research documents, whether they are published or not. The documents may come from teaching and research institutions in France or abroad, or from public or private research centers.
L'archive ouverte pluridisciplinaire HAL, est destinée au dépôt et à la diffusion de documents scientifiques de niveau recherche, publiés ou non, émanant des établissements d'enseignement et de recherche français ou étrangers, des laboratoires publics ou privés. 


\title{
Micromechanical modelling of the elastoplastic behaviour of metallic material under strain-path changes
}

\author{
Jamal Fajoui · David Gloaguen • Bruno Courant • \\ Ronald Guillén
}

\begin{abstract}
A two-level homogenization approach is applied for the micromechanical modelling of the elastoplastic material behaviour during various strain-path changes. A mechanical description of the grain is developed through a micro-meso transition based on a modified elastoplastic self-consistent approach which takes into account the dislocation evolution. Next, a meso-macro transition using a self-consistent model is used to deduce the macroscopic behaviour of the polycrystal. A correct agreement is observed between the simulations and the experimental results at the mesoscopic and macroscopic levels.
\end{abstract}

Keywords Plasticity - Dislocations - Microstructural · Homogenization · Self-consistent model $\cdot$ Non-local hardening

\section{Introduction}

During sheet metal forming, the material undergoes complex strain-path changes with large strains. These processes are often limited by damage, striction, structural instabilities or localized deformation (shear bands) [1]. Optimizing such technologies requires a good knowledge of the mechanical behaviour and an accurate modelling of the plastic anisotropic behaviour of deformed materials. The prediction of mechanical properties, in particular, work hardening is one of the most challenging exercises for material sciences. Many experimental studies have shown that changes in strain-path, which are usual in multiple-stage forming processes, can

J. Fajoui $(\varangle) \cdot$ D. Gloaguen · B. Courant · R. Guillén GeM, Institut de Recherche en Génie Civil et Mécanique, Université de Nantes, Ecole Centrale de Nantes,

CNRS UMR 6183, 37 Boulevard de l'Université, BP 406, 44602 Saint-Nazaire, France

e-mail: jamal.fajoui@univ-nantes.fr also lead to plastic anisotropy following the transition [2-6]. The transition region is often characterized by a change of the flow stress and the work hardening rate, which have significant influence on the formability of the material. This kind of anisotropy results from the evolution of the dislocation microstructure made up of cells (with a relatively low dislocation density) and walls (with a higher dislocation density) $[7,8]$.

The development of crystal plasticity models $[9,10]$, where the distribution of the crystal orientations in the given material and the geometry of the available slip systems, in the given crystals, are taken into account explicitly, opens the possibility of characterizing more accurately the mechanical behaviour using an adequate polycrystalline model (e.g. a Taylor model or a self-consistent scheme) [11-13]. The local behaviour (at the grain level) is based on the assumption of uniform deformation neglecting the formation of the dislocation microstructure. These approaches permit to predict correctly the yield surface, the crystallographic texture linked to the plastic deformation, the intergranular stresses due to the initial and induced anisotropy during monotonic loadings [14-17]. Nevertheless, this kind of description fails to reproduce the mechanical behaviour during changing strain paths [18]. These methods do not, or weakly, take account of the formation, the evolution and the stability of induced dislocation microstructures which are strongly path dependent.

Recently, several studies were performed to develop models which incorporate the effects of dislocation heterogeneities on the mechanical response of a metallic material (see for example Ref. [19-21]). The first attempt to model the dislocation microstructure was proposed by Mughrabi [22] who introduced a model in which the crystal is considered as a composite consisting of hard dislocation walls of high local dislocation density which are separated by soft regions of low local dislocation density. Peeters et al. [23] devel- 
oped a semi-phenomenological crystal plasticity model that incorporates more details of microstructural evolution at the grain scale (evolution of dislocation densities) and used a full-constraint Taylor model to describe the behaviour of b.c.c. polycrystals. Karaman et al. [24] have used a similar approach so as to model successfully the deformation of hadfield steel with a viscoplastic self-consistent scheme. Viatkina et al. [25] employed a micromechanical model of a dislocation cell structure accounting for the material inhomogeneity and incorporates the internal stresses in a physicalbased manner. A composite model is employed to describe the material with its dislocation cell structure. This approach was successfully applied to predict the macroscopic behaviour of f.c.c. alloys after various strain path changes. The concept of non-local hardening (the yield stress of the walls increases with the plastic strain of the cells) related to the two-phase cells structure was introduced, for the first time, by Muller et al. [26] and developed later by Lemoine et al. $[27,28]$ with a two-scale homogenization based on a polycrystaline model. The morphology of the dislocation structure is taken into account by the authors. The different numerical results concerning complex path loads seem to show a better description of the influence of the dislocation structure.

In a previous work [29], based on the approach proposed by Muller et al. [26,27], the present authors have extended the elasto-plastic self-consistent model (EPSC) by incorporating more details of the microstructure at the grain scale. More precisely, a micro-mechanical description of a single-crystal was developed through a micro-mesoscopic transition. The grain was considered as a two-phase material (dislocation walls and cells). Dislocation densities on each slip system were considered as internal variables and a hardening matrix, taking account of the different dislocations interactions, was proposed. Next, a meso-macroscopic transition, using the EPSC method [11], was applied to deduce the macroscopic response of the f.c.c. aggregate. The simulations based on the developed model demonstrated its capability to predict the experimental response of the observed material after moderate strain-path changes.

Nevertheless, a simple Kroner's approach was applied and adapted considering each grain as a two-phase material. This scheme considers the plastic incompatibility as a "stress free" strain [30] leading to pure elastic mechanical interactions. Consequently, the internal stresses are strongly overestimated since "plastic" accommodation around the inclusion (cell or wall) is neglected. Based on a useful (and crude) assumption of plastic accommodation proposed by Berveiller and Zaoui [31], a plastic accommodation factor $\alpha_{T}$ has been introduced. This factor has been used afterwards with an additional assumption concerning $\alpha_{T}$ : it was supposed to be independent of the plastic flow and $\alpha_{T}=1 / 10$. This approximation permitted to simulate the mechanical response of real metals with reasonable computer time. This approach has been taken on the grounds of simplicity. On the other side, this formulation cannot describe correctly the cell-wall interactions and must be taken as a convenient "first approximation' investigation.

The main objective of this paper is to enhance the twoscale transition model developed in Gloaguen et al. [29] with an improved description of the mesoscopic (at the grain level) elastoplastic behaviour with the more realistic selfconsistent (SC) approach. The attention is focused on the development of a model which incorporates with more accuracy the effects of dislocation heterogeneities on the mechanical response of a metallic material. The intragranular heterogeneities are taken into account by a new nonlocal work-hardening linked to the two-phase description. The expressions of the hardening laws and the local elastoplastic behaviour have been determined taking into account internal variables like dislocation densities on each deformation system, phase and volume fraction of the cells. The cell structure model has been enhanced by evolution equations for the dislocation density. In order to verify the model, different experimental results with complex loading paths on singlecrystals and polycrystals have been used here. In this paper, the two-scale transition approach is considered for f.c.c. and b.c.c. metals containing a cell structure.

\section{Two-level homogenization approach}

\subsection{Micro-meso transition scheme}

For obtaining the overall response of a heterogeneous medium, we used the average-field theory (or the mean-field theory). This theory is based on the fact that the effective mechanical properties measured in experiments are relations between the volume average of the strain and stress of microscopically heterogeneous samples. Hence, macrofields are defined as the volume averages of the corresponding microfields, and the effective properties are determined as relations between the averaged microfields. In our analysis, we consider single-phase polycrystals such as f.c.c. and b.c.c. metals. Based on the work of Mughrabi [22], each grain is treated as a two-phase composite consisting of cell walls of high dislocation density and cell interiors relatively poor in dislocations [7]. In each phase, it is assumed that slip is the predominant deformation mechanism and that Schmid's law is valid. The elastic behaviour of both phases is assumed to be the same. The one-site SC model (for more details, see [30-34]) is based on the solution of an ellipsoidal inclusion representing a given phase (cell walls or cells), embedded in a homogeneous equivalent medium (HEM). For the case of a two-phase composite, the inclusion represents cell interiors or cell walls and the HEM, the grain. The consistency conditions (global compatibility and equilibrium) require that the average of the local fields over the volume of the grain should 
be equal to the mesoscopically imposed ones. These averages represent the fields of the HEM. The SC formulation allows each phase to deform differently, according to its properties and depending on the strength of the interaction between the phase and its surroundings (via the Eshelby tensor). In this sense, each phase is in turn considered as an ellipsoidal inclusion surrounded by a HEM which have the average properties of the grain. It should be noticed that the phase state is characterized by the initial shape and orientation of the ellipsoid corresponding to a phase. The interaction between the inclusion and the HEM is solved by means of the Eshelby formalism. The HEM properties are not known in advance, but have to be calculated as the average of the individual phase behaviours, once convergence is achieved. The presented model is formulated with the assumption of small deformation theory.

Respectively describing the mean strain in the cells (c) and walls (w) by $\varepsilon^{c}$ and $\varepsilon^{w}$, the deformation $\varepsilon$ within the grain can be written from the usual rule of mixture by:

$\varepsilon=f_{w} \varepsilon^{w}+f_{c} \varepsilon^{c}$

where $f_{c}$ (respectively $f_{w}=1-f_{c}$ ) is the volume fraction of cell interiors (respectively cell walls) in a grain. Using this mixture rule for stress, one has:

$\sigma=\left(1-f_{c}\right) \sigma^{w}+f_{c} \sigma^{c}$

The slip systems are defined by the unit normal to the slip plane $n_{\alpha}^{g}$ (the subscript ' $\alpha$ ' stands for walls ' $w$ ' and cells 'c') and the slip direction $m_{\alpha}^{g}$. If $\dot{\gamma}_{\alpha}^{g}$ denotes the slip rate on active system $g$, the plastic strain rate $\dot{\varepsilon}^{p \alpha}$ is given by:

$\dot{\varepsilon}^{p \alpha}=\sum_{g} R_{\alpha}^{g} \dot{\gamma}_{\alpha}^{g}$

$R_{\alpha}^{g}$ is the Schmid tensor.

With progressing strain, a dislocation cellular arrangement expands, composed of cell walls with high dislocation density which enclose cell interiors of low dislocation density. Dislocation sources, inside the material, generate mobile dislocations which interact with dislocations in the cell walls and increase dislocation density in this region. Few dislocations accumulate within cells. The annihilation of dislocations is mainly localized inside walls due to the rearrangement of dislocations associated with the plastic strain inside walls [34]. Finally, the plastic glide in cells increases dislocations and the critical shear stress in the walls. This is the non-local hardening concept introduced by Muller et al. [26].

In the general case, the non-local hardening laws can be written for a two-phase composite as follows [29]:

$$
\begin{aligned}
\dot{\tau}_{c r c}^{g} & =\sum_{h} H_{c c}^{g h} \dot{\gamma}_{c}^{h}+\sum_{h} H_{c w}^{g h} \dot{\gamma}_{w}^{h} \text { and } \dot{\tau}_{c r w}^{g} \\
& =\sum_{h} H_{w c}^{g h} \dot{\gamma}_{c}^{h}+\sum_{h} H_{w w}^{g h} \dot{\gamma}_{w}^{h}
\end{aligned}
$$

$\dot{\tau}_{c r c}^{g}$ and $\dot{\tau}_{c r w}^{g}$ denote the critical resolved shear stresses (CRSS) rates within the cells and the walls. $H_{c c}^{g h}, H_{c w}^{g h}, H_{w c}^{g h}$ and $H_{w w}^{g h}$ are the hardening matrices describing interactions between dislocations moving on various slip systems. For an appropriate representation of the hardening behaviour, these matrices are expressed with microstructural variables such as dislocation density. The constitutive laws of dislocation evolution are generalised in a density context. The equations are formulated to describe the average behaviour of a large number of dislocations in a 'continuum' material point. The following evolution equations for the dislocation density in metals, are used [35]:

$\dot{\rho}_{w}^{g}=\dot{\rho}_{w}^{g+}+\dot{\rho}_{w}^{g-}$

with:

$\dot{\rho}_{w}^{g+}=\frac{1}{b L^{g}} \dot{\gamma}_{c}^{g} \quad$ and $\quad \dot{\rho}_{w}^{g-}=-\frac{2 y_{c}}{b} \rho_{w}^{g} \dot{\gamma}_{w}^{g}$

$\dot{\rho}_{w}^{g+}$ corresponds to the rate of increase of dislocation density inside the walls on the system $g$. This term is responsible for the creation and the development of the cell structure. This flux consists of dislocations that glide towards walls through the applied stress. $\dot{\rho}_{w}^{g-}$ is the annihilation term which takes into account the dynamic recovery during the deformation. $b$ is the magnitude of the Burgers vector and $y_{c}$ is proportional to the annihilation distance of dislocation dipoles. $L^{g}$ is the mean free path of mobile dislocations in the cell interiors associated to the deformation system $g$. Its evolution is governed by the density of point obstacles (essentially cell walls) cutting the system $g$ and creating interactions with gliding dislocations. Therefore:

$L^{g}=k_{L}{\sqrt{\sum_{l \neq g} \rho_{w}^{l}}}^{-1}$

where $k_{L}$ is a material constant.

The density of immobile dislocations within the cell $\rho_{c}^{g}$ is assumed to be constant with the deformation.

The CRSS can be related to the dislocation densities by the hardening relation [36]:

$\tau_{c r \alpha}^{g}=\tau_{c r 0 \alpha}^{g}+\xi \mu b \sqrt{\sum_{h} a^{g h} \rho_{\alpha}^{h}}$

where $\tau_{c r 0 \alpha}^{g}$ is the initial reference shear stress on system $g$, $\xi$ is a constant depending on the interaction of dislocations $(\xi \in[0.25-0.6])$. According to Franciosi et al. [36], $a^{g h}$ is a hardening matrix which terms depend on the type of interactions between dislocation families $g$ and $h$. Only two terms, $a^{g g}$ and $a_{g \neq h}^{g h}$ (respective self- and cross-hardening parameters), will be considered. $\rho_{\alpha}^{h}$ is the dislocation density in the phase $\alpha$. 
Differentiating Eq. 8 with respect to time and considering Eqs. 6 and 7, the work-hardening matrices $H_{w w}^{g h}$ and $H_{w c}^{g h}$ are:

$H_{w c}^{g h}=\frac{\xi^{2} \mu^{2} b}{2\left(\tau_{c r w}^{g}-\tau_{c r 0 w}^{g}\right)} \frac{a^{g h}}{L^{h}}$

$H_{w w}^{g h}=-\frac{y_{c} \xi^{2} \mu^{2} b}{\left(\tau_{c r w}^{g}-\tau_{c r 0 w}^{g}\right)} \rho_{w}^{h} a^{g h}$

Because of the low accumulation of dislocations inside cells, $H_{c c}^{g h}$ is simply described by a constant. The influence of walls on the evolution of dislocations in cells can be considered as negligible. Finally:

$H_{c c}^{g h}=\frac{\varsigma}{\tau_{c r c}^{g}-\tau_{c r 0 c}^{g}} a^{g h}$

$H_{c w}^{g h}=0$

where $\zeta$ is a constant.

During the plastic regime, the variation of the cell interior volume fraction for a grain $I$ is considered as a function of the accumulated slip through the following empiric relation [28]:

$f_{c I}=f_{\infty}+\left(f_{0}-f_{\infty}\right) \exp \left(-\frac{\gamma_{c I}^{\text {acc }}}{\gamma^{\text {par }}}\right)$

where $f_{0}$ is the initial volume fraction and $f_{\propto}$ the saturation value at large strains. $\gamma^{\mathrm{par}}$ is a parameter which describes the decrease rate of $f_{c I} \cdot \gamma_{c I}^{\text {acc }}$ is the accumulated slip in the cell in the grain $I$. This relation allows to take the intergranular plastic heterogeneity into account. The volume fraction of cells for each crystallite develops differently, in relation to the effect of the grain orientation. It only depends on the plasticity activity. In the remainder of the paper, the cell volume fraction will be denoted $f_{c}$ instead of $f_{c I}$.

Following Schmid's law, the plastic glide on a slip system $g$ is possible if the resolved shear stress $\tau_{\alpha}^{g}$ reaches a critical value $\tau_{c r \alpha}^{g}$ which depends on the hardening state of the slip system. Since this necessary condition is insufficient, a complementary condition has to be checked simultaneously: the increment (rate) of the resolved shear stress must be equal to the increment of the critical one. This can be written on the form:

$\tau_{\alpha}^{g}=R_{\alpha}^{g} . . \sigma^{\alpha}$ and $\dot{\tau}_{\alpha}^{g}=R_{\alpha}^{g} . . \dot{\sigma}^{\alpha}$

where $A . . B$ denotes the double scalar product $A_{i j k l} B_{k l t u}$ using the Einstein summation convention. The main problem with the EPSC model is to determine which combination of slip systems will actually be activated at each step of the plastic deformation path. In this case, all the possible combinations of potentially active systems must be scanned to find one that satisfies the two previous (Eq. 14) conditions simultaneously. Because this must be done for the two phases of each grain, running time considerations become the main task of the model. Recently, Ben Zineb et al. [37] have proposed a new formulation. This one was extended to the polycrystalline model framework by Lorrain et al. [38]. The accuracy of the simulations was also evaluated at the mesoscopic and the macroscopic levels by referring to mechanical experiments (tensile tests, neutron or X-ray diffraction) [13]. The main challenge in the present context is to adapt this formulation to the case of a two-phase composite with a non-local hardening behaviour. Based on the work of Ben Zineb et al. and the Eq. (4), the slip rate on a system $g$ for each phase can be expressed with the following equations:

$\left\{\begin{array}{l}\dot{\gamma}_{c}^{g}=K_{c c}^{g} \dot{\tau}_{c}^{g}+K_{c w}^{g} \dot{\tau}_{w}^{g} \\ \dot{\gamma}_{w}^{g}=K_{w c}^{g} \dot{\tau}_{c}^{g}+K_{w w}^{g} \dot{\tau}_{w}^{g}\end{array}\right.$

$K_{c c}^{g h}$ and $K_{w w}^{g h}$ are given by:

$\left\{\begin{aligned} K_{c c}^{g}= & \frac{1}{H_{c c}^{g}}\left[\frac{1}{2}\left(1+\tanh \left(\tau_{c}^{g}\right)\right)\right] \\ & \times\left[\frac{1}{2}\left(1+\tanh \left(k\left(\frac{\tau_{c}^{g}}{\tau_{c r c}^{g}}-1\right)\right)\right)\right]\left[\frac{1}{2}\left(1+\tanh \left(\dot{\tau}_{c}^{g}\right)\right)\right] \\ K_{w w}^{g}= & \frac{1}{H_{w w}^{g}}\left[\frac{1}{2}\left(1+\tanh \left(\tau_{w}^{g}\right)\right)\right] \\ & \times\left[\frac{1}{2}\left(1+\tanh \left(k\left(\frac{\tau_{w}^{g}}{\tau_{c r w}^{g}}-1\right)\right)\right)\right]\left[\frac{1}{2}\left(1+\tanh \left(\dot{\tau}_{w}^{g}\right)\right)\right]\end{aligned}\right.$

where $k$ is a material constant [39].

Since the influence of walls on the evolution of dislocations in cells is negligible, $K_{c w}^{g}$ is assumed to be equal to 0 . The last parameter $K_{w c}^{g}$, corresponding to the non-local contribution from the cells to the walls, is defined by:

$$
\begin{aligned}
K_{w c}^{g}= & \frac{\kappa}{H_{c c}^{g}}\left[\frac{1}{2}\left(1+\tanh \left(\tau_{c}^{g}\right)\right)\right] \\
& \times\left[\frac{1}{2}\left(1+\tanh \left(k\left(\frac{\tau_{c}^{g}}{\tau_{c r c}^{g}}-1\right)\right)\right)\right] \\
& \times\left[\frac{1}{2}\left(1+\tanh \left(\dot{\tau}_{c}^{g}\right)\right)\right]
\end{aligned}
$$

The parameter $\kappa(\kappa<1)$ quantifies the strength of the nonlocal hardening and consequently the influence of the cells on the walls. This parameter depends on the studied material (b.c.c or f.c.c), the initial dislocation microstructure and the mechanical test.

Using the relations (14) and (15), the slip rate on a system $g$, for the cell and wall regions, becomes:

$\left\{\begin{array}{l}\dot{\gamma}_{c}^{g}=K_{c c}^{g} R_{c}^{g} . . \dot{\sigma}^{c}+K_{c w}^{g} R_{w}^{g} . . \dot{\sigma}^{w} \\ \dot{\gamma}_{w}^{g}=K_{w c}^{g} R_{c}^{g} . . \dot{\sigma}^{c}+K_{w w}^{g} R_{w}^{g} . . \dot{\sigma}^{w}\end{array}\right.$ 
By using the usual generalized Hooke's law, the stress rate for each phase is obtained:

$$
\dot{\sigma}^{\alpha}=c . .\left(\dot{\varepsilon}^{\alpha}-\dot{\varepsilon}^{p \alpha}\right)
$$

$c$ is the elastic constant tensor at the grain level. Using the relations (18) and (19) the slip rate for each phase is given by:

$$
\left\{\begin{aligned}
\dot{\gamma}_{c}^{g}= & K_{c c}^{g} R_{c}^{g} . . c . .\left(\dot{\varepsilon}^{c}-\sum_{h} R_{c}^{h} \dot{\gamma}_{c}^{h}\right) \\
& +K_{c w}^{g} R_{w}^{g} . . c . .\left(\dot{\varepsilon}^{w}-\sum_{h} R_{w}^{h} \dot{\gamma}_{w}^{h}\right) \\
\dot{\gamma}_{w}^{g}= & K_{w c}^{g} R_{i j}^{c g} . . c . .\left(\dot{\varepsilon}^{c}-\sum_{h} R_{c}^{h} \dot{\gamma}_{c}^{h}\right) \\
& +K_{w w}^{g} R_{w}^{g} . . c . .\left(\dot{\varepsilon}^{w}-\sum_{h} R_{w}^{h} \dot{\gamma}_{w}^{h}\right)
\end{aligned}\right.
$$

After some calculations, one obtains the following matrix form:

$$
\begin{aligned}
& \left(\begin{array}{cc}
\left(\delta_{g h}+K_{c c}^{g} R_{c}^{g} . . c . . R_{c}^{h}\right) & \left(K_{c w}^{g} R_{w}^{g} . . c . . R_{w}^{h}\right) \\
\left(K_{w c}^{g} R_{c}^{g} . . c . . R_{c}^{h}\right) & \left(\delta_{g h}+K_{w w}^{g} R_{w}^{g} . . c . . R_{w}^{h}\right)
\end{array}\right)\left(\begin{array}{c}
\dot{\gamma}_{c}^{h} \\
\dot{\gamma}_{w}^{h}
\end{array}\right) \\
& =\left(\begin{array}{l}
K_{c c}^{g} R_{c}^{g} . . c . . \dot{\varepsilon}_{k l}^{c}+K_{c w}^{g} R_{w}^{g} . . c . . \dot{\varepsilon}^{w} \\
K_{w c}^{g} R_{c}^{g} . . c . . \dot{\varepsilon}^{c}+K_{w w}^{g} R_{w}^{g} . . c . . \dot{\varepsilon}^{w}
\end{array}\right)
\end{aligned}
$$

where $\delta_{g h}$ is the Kronecker's delta. It should be noticed that the subscripts $\mathrm{g}$ and $\mathrm{h}$ vary from one to $N(N$ :number of active systems for each phase. $N=12$ for f.c.c and 24 for b.c.c metals) for each term of the expression (21). Developing (21), the slip rate is given by:

$$
\begin{aligned}
\left(\begin{array}{c}
\dot{\gamma}_{c}^{g} \\
\dot{\gamma}_{w}^{g}
\end{array}\right)= & \left(\begin{array}{cc}
M_{c c}^{g h} & M_{c w}^{g h} \\
M_{w c}^{g h} & M_{w w}^{g h}
\end{array}\right) \\
& \times\left(\begin{array}{c}
K_{c c}^{h} R_{c}^{h} . . c . . \dot{\varepsilon}^{c}+K_{c w}^{h} R_{w}^{h} . . c . . \dot{\varepsilon}^{w} \\
K_{w c}^{h} R_{c}^{h} . . c . . \dot{\varepsilon}^{c}+K_{w w}^{h} R_{w}^{h} . . c . . \dot{\varepsilon}^{w}
\end{array}\right)
\end{aligned}
$$

where the matrix $M_{\alpha \beta}^{g h}$ is given by the following equation:

$$
\begin{aligned}
& \left(\begin{array}{cc}
M_{c c}^{g h} & M_{c w}^{g h} \\
M_{w c}^{g h} & M_{w w}^{g h}
\end{array}\right) \\
& =\left(\begin{array}{cc}
\left(\delta_{g h}+K_{c c}^{g} R_{c}^{g} . . c . . R_{c}^{h}\right) & \left(K_{c w}^{g} R_{w}^{g} . . c . . R_{w}^{h}\right) \\
\left(K_{w c}^{g} R_{c}^{g} . . c . . R_{c}^{h}\right) & \left(\delta_{g h}+K_{w w}^{g} R_{w}^{g} . . c . . R_{w}^{h}\right)
\end{array}\right)^{-1}
\end{aligned}
$$

One can deduce the expression of the stress rate tensors:

$$
\begin{aligned}
\dot{\sigma}^{c}= & \left(c-c . .\left(\sum_{\mathrm{g}} R_{c}^{g} M_{c c}^{g h} K_{c c}^{h} R_{c}^{h} . . c\right)\right. \\
& \left.-c . .\left(\sum_{\mathrm{g}} R_{c}^{g} M_{c w}^{g h} K_{w c}^{h} R_{c}^{h} . . c\right)\right) . . \dot{\varepsilon}^{c} \\
& +\left(-c . .\left(\sum_{\mathrm{g}} R_{c}^{g} M_{c c}^{g h} K_{c w}^{h} R_{w}^{h} . . c\right)\right. \\
& \left.-c . .\left(\sum_{\mathrm{g}} R_{c}^{g} M_{c w}^{g h} K_{w w}^{h} R_{w}^{h} . . c\right)\right) . . \dot{\varepsilon}^{w} \\
\dot{\sigma}^{w}= & \left(-c . .\left(\sum_{\mathrm{g}} R_{w}^{g} M_{w c}^{g h} K_{c c}^{h} R_{c}^{h} . . c\right)\right. \\
& \left.-c . .\left(\sum_{\mathrm{g}} R_{w}^{g} M_{w w}^{g h} K_{w c}^{h} R_{c}^{h} . . c\right)\right) . . \dot{\varepsilon}^{c} \\
& +\left(c-c . .\left(\sum_{\mathrm{g}} R_{w}^{g} M_{w c}^{g h} K_{c w}^{h} R_{w}^{h} . . c\right)\right. \\
& \left.-c . .\left(\sum_{\mathrm{g}} R_{w}^{g} M_{w w}^{g h} K_{w w}^{h} R_{w}^{h} . . c\right)\right) . . \dot{\varepsilon}^{w}
\end{aligned}
$$

Using (24), we can write again the stress tensors in a condensed form as following:

$\dot{\sigma}^{c}=\ell^{c c} . . \dot{\varepsilon}^{c}+\ell^{c w} . . \dot{\varepsilon}^{w}$ and $\dot{\sigma}^{w}=\ell^{w c} . . \dot{\varepsilon}^{c}+\ell^{w w} .{ }_{.} \dot{\varepsilon}^{w}$

where $\ell^{c c}, \ell^{c w}, \ell^{w c}$ et $\ell^{w w}$ are the microscopic tangent moduli tensors corresponding to a two-phase non-local material. These tensors depend on the local and non-local hardening matrices which are expressed explicitly with microstructural variables like dislocation density. However, $H_{c w}^{g h}=0, K_{c w}^{g h}=$ 0 and $M_{c w}^{g h}=0$, the microscopic tangent moduli tensors simplified to:

$$
\left\{\begin{aligned}
\ell^{c c}= & c_{-} c . .\left(\sum_{g} R_{c}^{g} M_{c c}^{g h} K_{c c}^{h} R_{c}^{h} . . c\right) \\
\ell^{c w}= & 0 \\
\ell^{w c}= & -c . .\left(\sum_{g} R_{w}^{g} M_{w c}^{g h} K_{c c}^{h} R_{c}^{h} . . c\right) \\
& -c . .\left(\sum_{g} R_{w}^{g} M_{w w}^{g h} K_{w c}^{h} R_{c}^{h} . . c\right) \\
\ell^{w w}= & c-c . .\left(\sum_{g} R_{w}^{g} M_{w w}^{g h} K_{w w}^{h} R_{w}^{h} . . c\right)
\end{aligned}\right.
$$

The stress rates at the microscopic and mesoscopic scales are linked by:

$\dot{\sigma}=f_{c} \dot{\sigma}^{c}+\left(1-f_{c}\right) \dot{\sigma}^{w}$ 
Using (25) and (27) and after some calculations, one can find:

$$
\begin{aligned}
\dot{\sigma}= & \left(f_{c} \ell^{c c}+\left(1-f_{c}\right) \ell^{w c}\right) . . \dot{\varepsilon}^{c} \\
& +\left(f_{c} \ell^{c w}+\left(1-f_{c}\right) \ell^{w w}\right) . . \dot{\varepsilon}^{w}=\ell . . \dot{\varepsilon}
\end{aligned}
$$

The tangent properties of the single-crystal are given by the tensor $\ell$.

In what follows, we assume the existence of a strain rate localization tensor linking each phase's mean strain rate with the corresponding grain's one:

$$
\left\{\begin{array}{l}
\dot{\varepsilon}^{c}=a^{c} . . \dot{\varepsilon} \\
\dot{\varepsilon}^{w}=a^{w} . . \dot{\varepsilon}
\end{array}\right.
$$

These tensors are given by:

$$
\left\{\begin{array}{l}
a^{c}=\left(I+s^{e s h} . . \ell^{-1} . . \Delta \ell^{c c}\right)^{-1} \\
a^{w}=\left(I+s^{e s h} . . \ell^{-1} . . \Delta \ell^{w w}\right)^{-1} . .\left(I-s^{e s h} . . \ell^{-1} . . \ell^{w c} . . a^{c}\right)
\end{array}\right.
$$

where $I$ is the fourth order identity tensor, $\Delta \ell^{\alpha \alpha}=\ell^{\alpha \alpha}-\ell$ and $s^{e s h}$, the classical Eshelby tensor at the grain scale. This tensor takes into account the effect of inclusion morphology upon the average plastic crystal behaviour. Replacing the expressions of $\dot{\varepsilon}^{\alpha}$ into (28), the stress tensor at the mesoscopic scale is given by:

$$
\begin{aligned}
\dot{\sigma}= & \left(\left(f_{c} \ell^{c c}+\left(1-f_{c}\right) \ell^{w c}\right) . . a^{c}\right. \\
& \left.+\left(f_{c} \ell^{c w}+\left(1-f_{c}\right) \ell^{w w}\right) . . a^{w}\right) . . \dot{\varepsilon}
\end{aligned}
$$

with:

$$
\begin{aligned}
\ell= & \left(f_{c} \ell^{c c}+\left(1-f_{c}\right) \ell^{w c}\right) . . a^{c} \\
& +\left(f_{c} \ell^{c w}+\left(1-f_{c}\right) \ell^{w w}\right) . . a^{w}
\end{aligned}
$$

The tensor $\ell$ has a complex form in the two-phase composite framework compared to the pure single crystal constitutive relation [39]. It depends on active systems, elastic properties, inclusion morphology, stress rate and deformation history of each phase. The Eq. (32) is a non-linear implicit equation which is solved by iteration. This model involves calculating the tensor $\ell$ once for each grain in each deformation increment, rather than just once per increment in the 'classic' EPSC model, and is therefore much more expensive in computer time.

\subsection{Meso-macro transition scheme}

To complete the two-scale transition model, the overall constitutive relationship for the polycrystal must be determined. It can be deduced from Eq. (32) using homogenization procedures. In this paper a SC approach is also adopted to perform this homogenization (for more details, see [10,11,14, 30-34]). Figure 1 illustrates the two-stage SC model developed in this work.

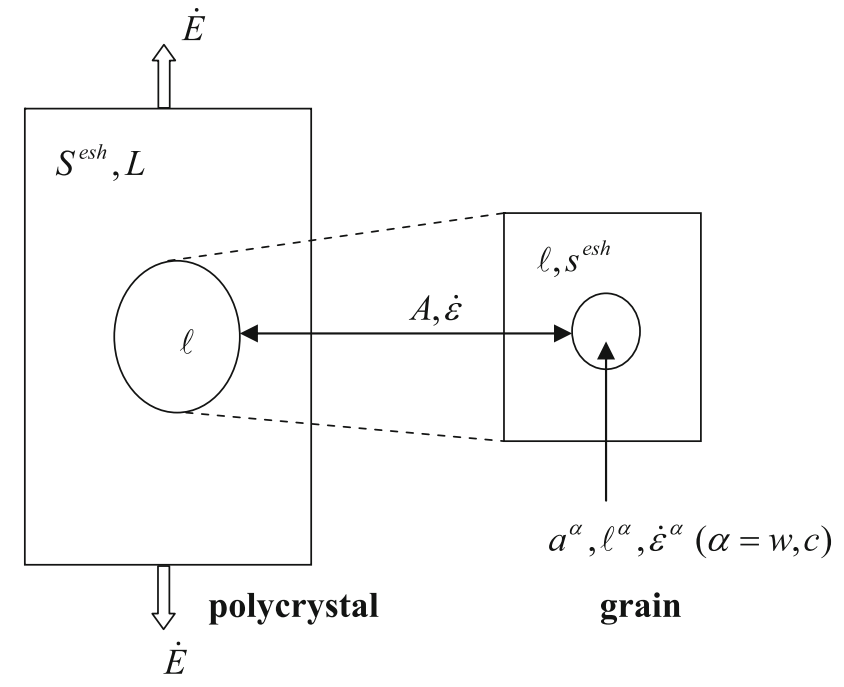

Fig. 1 Schematic diagram showing the two-stage self-consistent model within each grain and within the material. The symbols are defined in the text

Using $L$, the macroscopic tangent moduli tensor, the global behaviour of the material can be described as:

$\dot{\Sigma}=L . . \dot{E}$

where $\dot{E}$ and $\dot{\Sigma}$ are the overall strain rate and stress rate on the material.

The strain rate at the mesoscopic and macroscopic scales are linked by a localization tensor $A$ :

$\dot{\varepsilon}=A . . \dot{E}=\left[I+S^{e s h} . . L^{-1} . .(\ell-L)\right]^{-1} . . \dot{E}$

where $\mathrm{S}^{e s h}$ is the Eshelby tensor at the macroscopic scale. It takes into account the interaction between grains and the homogeneous equivalent medium. $\ell$ corresponds to the modulus defined in Sect. 2.1. After some calculations one can obtain:

$\dot{\sigma}=\ell . . \dot{\varepsilon}=\ell . .\left[I+S^{e s h} . . L^{-1} . .(\ell-L)\right]^{-1} . . \dot{E}$

The volume averages of the local stress and strain tensors must coincide with the overall strain and stress. These conditions give the tensor $\mathrm{L}$ as a weighted average of the mesoscopic tensor $\ell$ :

$L=\left\langle\ell . .\left[I+S^{e s h} . . L^{-1} . .(\ell-L)\right]^{-1}\right\rangle$

Like Eqs. 32, 36 is a non-linear implicit equation. This equation can be solved by iteration. Once $L$ is known, by specifying an overall stress or strain increment, the model can give the corresponding stress and strain tensors for each orientation. Therefore, the mechanical response of the polycrystal can be completely described at the different levels of the polycrystal. 


\section{Numerical analysis}

\subsection{Micro-meso transition scheme}

The performance of the enhanced model is verified here for single-crystals and polycrystals metals submitted to complex load paths. The experiment results used in this work have been published by research groups, on the basis of mechanical tests measurements [40-42]. In order to perform numerical simulations, the model parameters must be identified. Isotropic and homogenous elasticity, described by shear modulus $\mu$ and Poisson's ratio $\nu$, is assumed. A value of 0.5 for $\xi$ (Eq. 8) reflects multiple slip, involving the mutual intersection of dislocations of different slip systems. The cells are assumed to be spherical. The geometry of the cells is fixed during deformation whereas the dislocation densities will evolve (Eq. 5). The initial texture is taken isotropic. It is described by three randomly generated Euler's angles. Lattice rotation and thus texture changes are incorporated in the model. The model calculations were performed for a set of 100 spherical inclusions. This number of crystallites has been chosen to simulate the polycrystalline aggregate in an efficient way. For f.c.c. metals, the plastic deformation was modelled assuming $\{110\}\langle 111\rangle$ slip systems. In the case of b.c.c. materials, the slip occurs on $\{112\}\langle 111\rangle$ and $\{110\}\langle 111\rangle$ systems. The same initial CRSS $\tau_{c r 0}^{\alpha}$ is taken for each deformation mode. These CRSS are determined to reproduce the experimental yield stress. Only very few experimental results on the dislocation evolution is available. First, these values of parameters are chosen to be coherent with those given in the literature. After that, these parameters are adjusted so that the model gives a good fitting with the macroscopic experimental curve under monotonic deformation. This is justified since the parameters $\rho_{0}, y_{c}$ (Eq. 6), $k_{L}$ (Eq. 7), $\varsigma$ (Eq. 11), and $\kappa$ (Eq. 17) define the evolution of the dislocation density and the hardening behaviour. Therefore, they can be derived from the macroscopic behaviour. To determine the volume fraction, typical values for cell regions observed at small to moderate deformation, are adopted [3]. The $k$ value (Eq. 16) for each material is determined by comparing with a classic EPSC model under monotonic deformation (in term of main active deformation systems, crystal reorientation).

\subsection{Results of the micromechanical modelling}

In this section, numerical results are presented in the case of monotonic and sequential loadings for single-crystals and polycrystals.

\subsubsection{Monotonic loading}

In order to validate the elasto-plastic single-crystal constitutive law, the numerical results are compared with differ- ent experimental results obtained by Pollnow et al. [40] and Keh et al. [41]. The used material is an $\alpha$-iron single-crystal submitted to uniaxial tensions [40] and shear tests [41] with different loading directions. For each case, the identification procedure is made with a particular crystallographic orientation (Figs. 2a and 3a). For the model verification, the material parameters are constant for the other crystallographic orientations (Euler angles are given in each figure). Table 1 gives the material parameters used for each set of mechanical tests. The elastic behaviour is considered as isotropic.

Figures 2 and 3 show the simulation results for the monotonic tensions and shear tests and also the evolution of the internal stresses in cells and walls simulated. In general, the simulated results show that the major features in experimental stress-strain curves can be qualitatively captured. Figure $2 \mathrm{c}$,d show a slightly difference between the simulation results and the experimental data. In author's opinion, this difference seems to be due that the critical initial resolved shear stress which is taken the same for each slip system family. Introducing a difference between those families have been experimentally already enlightened [43] and used by Lorrain et al. [38], for example, to make a comparison to the work of Rauch [44]. In Fig. 3b,d, disparity between the model and experimentally response is observed. It is probably due to the assumption of small deformation theory. Several improvements of the presented approach are necessary. For example, the description using large-scale transformations is required. Nevertheless, this comparison shows the qualitative capabilities of the present approach.

Due to the accumulation of dislocations within walls (nonlocal hardening effects), the internal stress inside walls is equal to approximately four times the macroscopic yield stress. The dislocation density within walls is much higher than the cells one. The relative deviation, for the reference orientation, varies between 0 and $10 \%$. For the other cases, the maximum value reaches $20 \%$.

\subsubsection{Strain-path changes}

The experimental data are extracted from Keh et al. [41] on five specimens. The results presented in this part correspond to a shear prestrain followed by an unloading and a subsequent shear test performed in another direction. The studied material is the same as before but not at the same temperature; it explains why some parameters are different from the previous part. In order to validate the model, the same procedure is adopted. Table 2 gives the parameters identified from the Fig. $4 a$. Figure $4 b-d$ give the simulation results obtained with the parameters specified in the Table 2. One can note an agreement between the experimental data and the numerical results, except on the Fig. 4c. In this case, the model underestimates the experimental curve. The change of the hardening rate at $10 \%$ total strain during 
Fig. 2 Experimental and simulated results for monotonic tensile tests: a identifying parameters, $\mathbf{b}, \mathbf{c}$ and $\mathbf{d}$ model check

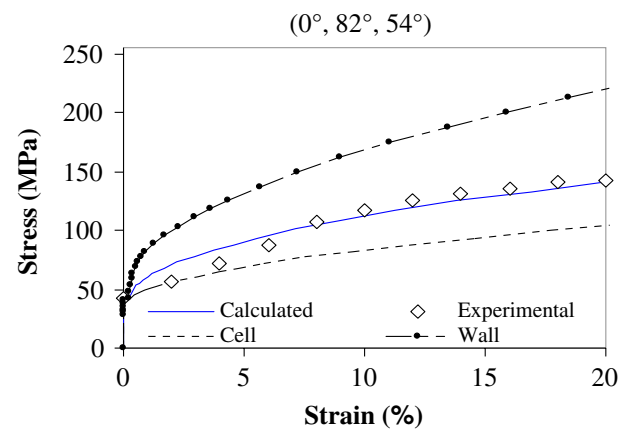

(a)

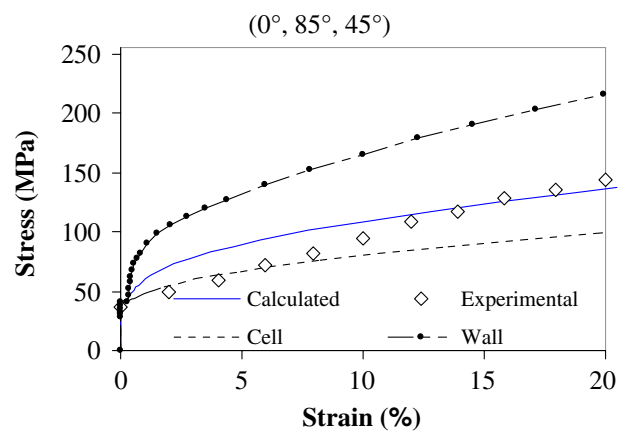

(c)

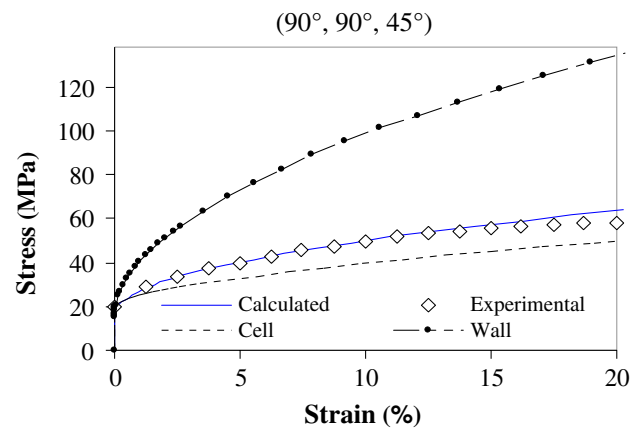

(a)

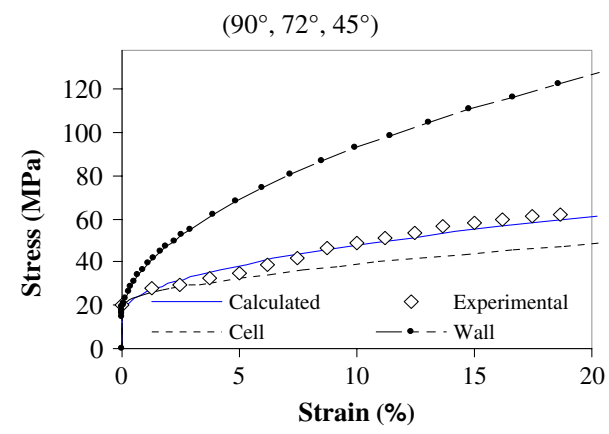

(c)

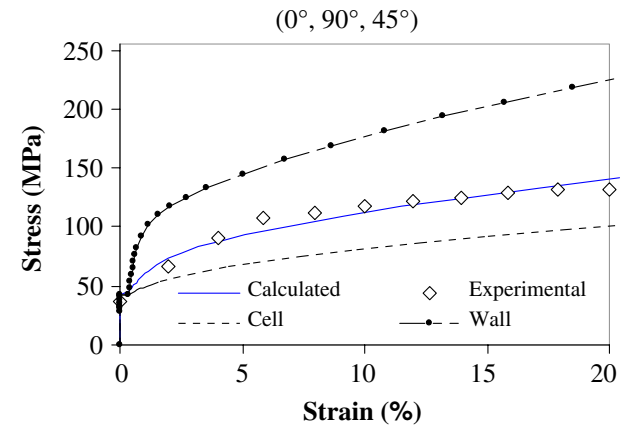

(b)

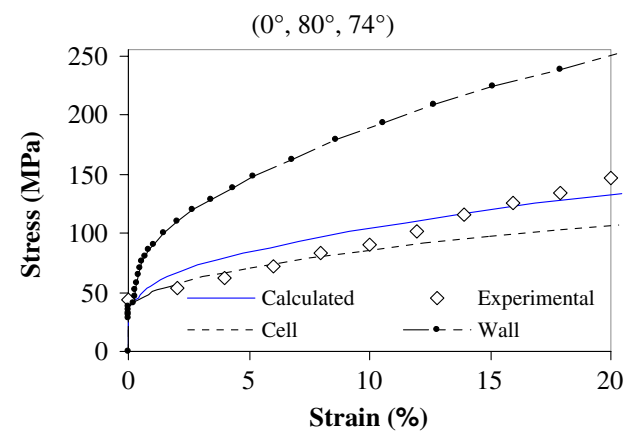

(d)

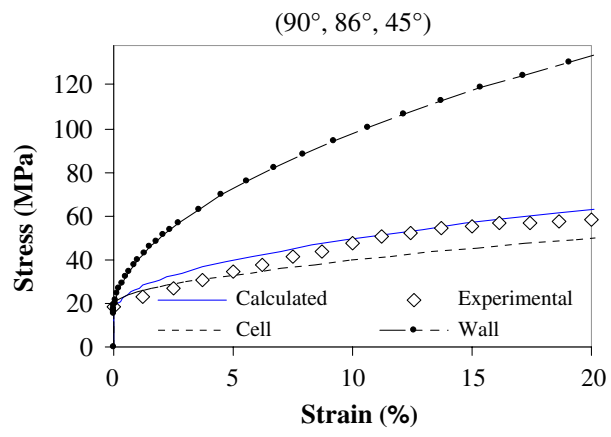

(b)

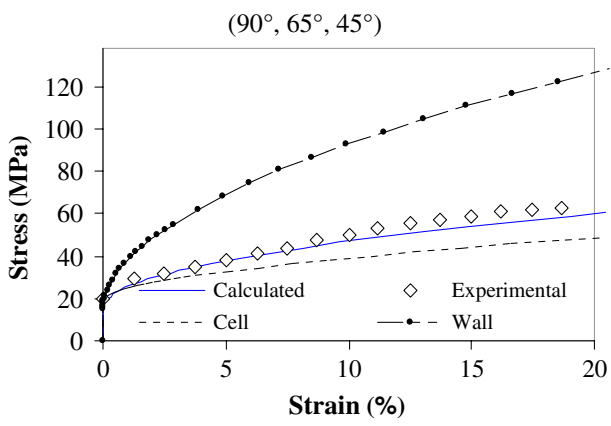

(d) the second loading is not captured by the model. Some discrepancy between the experiments and the simulations can be observed. A better agreement could be expected with more complex and sophisticated dislocation evolution laws.
Nevertheless, the present model is a reasonable compromise between complexity and accuracy with a limited number of parameters. From the results shown in Figs. 2, 3 and 4, it is clear that the crystal plasticity theory can be used to 
Table 1 Material constants for $\alpha$-iron

\begin{tabular}{llllll}
\hline$c_{11}(\mathrm{MPa})$ & $c_{12}(\mathrm{MPa})$ & $c_{44}(\mathrm{MPa})$ & $b(\mathrm{~m})$ & $\rho_{0}\left(\mathrm{~m}^{-2}\right)$ & $y_{c}(\mathrm{~m})$ \\
\hline 245 & 105 & 70 & $5 \times 10^{-9}$ & $10^{9}$ & $2.8 \times 10^{-9}$
\end{tabular}

accurately characterize the mechanical behaviour of cubic materials.

\subsubsection{Change of loading path in f.c.c. polycrystalline material}

The experimental results reported by Choteau et al. [42] for tension-compression tests on an AISI 316L austenitic stainless steel is used here to evaluate the capacity of the model to simulate correctly the different physical aspects of the polycrystal behaviour and the induced microstructure during complex load paths. The simulation results are compared with the experimental data for different pre-strain in ten- sion: 1, 2, 3 and $4 \%$ for the first test. The input texture consisted of a set of 100 equally-weighted lattice orientations representing a random texture. The grains were assumed to be equiaxed. Since simulations are conducted until moderate strains, no update shape of the grains was considered. There were not any available experimental data, in the literature, concerning crystallographic and morphologic textures of the tested materials. Nevertheless, it is clear that the influence of crystallographic texture is negligible compared to the effects of internal stress state introduced by the preloading. The elastic behaviour is considered isotropic (Table 3). The material parameters have been determined by fitting the experimental monotonic tensile curve (Fig. 5). The parameters values are listed in Table 4. A good correlation is obtained with the reference data. Figure 5 also shows the internal mechanical states within cells and walls $(\alpha=c, w)$ :

$\sigma_{11}^{\alpha}=\sum_{I} f_{I} \sigma_{11}^{I \alpha} \quad$ and $\quad \varepsilon_{11}^{\alpha}=\sum_{I} f_{I} \varepsilon_{11}^{I \alpha}$

Table 2 Model parameters for single crystal

\begin{tabular}{lccccccccccc}
\hline & $a^{g g}$ & $a^{g h}$ & $\begin{array}{l}\tau_{c r 0(112)} \\
(\mathrm{MPa})\end{array}$ & $\begin{array}{l}\tau_{c r 0(110)} \\
(\mathrm{MPa})\end{array}$ & $k_{L}$ & $f_{0}$ & $f_{\infty}$ & $\gamma^{\text {par }}$ & $\xi$ & $k$ \\
\hline Tensile test & 1 & 1.1 & 18 & 16 & 55 & 0.8 & 0.75 & 3.2 & 0.5 & 4.3 & 0.4 \\
Shear test & 1 & 1.1 & 17 & 15 & 55 & 0.86 & 0.75 & 3.2 & 0.5 & 4.3 & 0.4 \\
Sequential loading & 1 & 1.1 & 6 & 5 & 55 & 0.85 & 0.75 & 3.2 & 0.5 & 20 & 0.4 \\
\hline
\end{tabular}

Fig. 4 Experimental and simulated results for sequential loading tests: a identifying parameters, $\mathbf{b}, \mathbf{c}$ and $\mathbf{d}$ model check

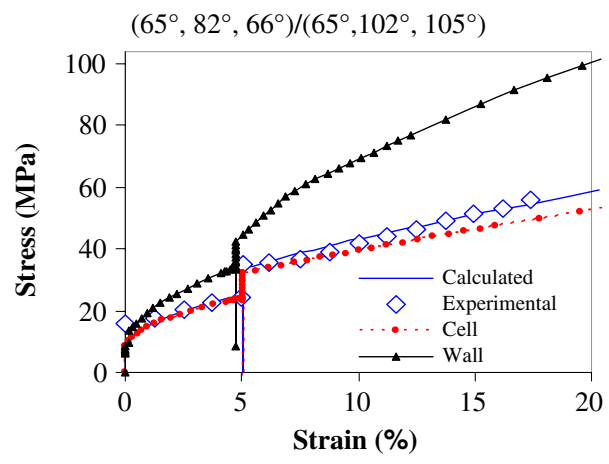

(a)

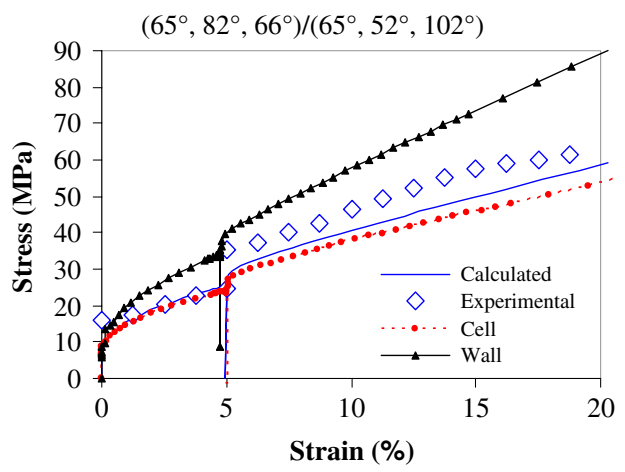

(c)

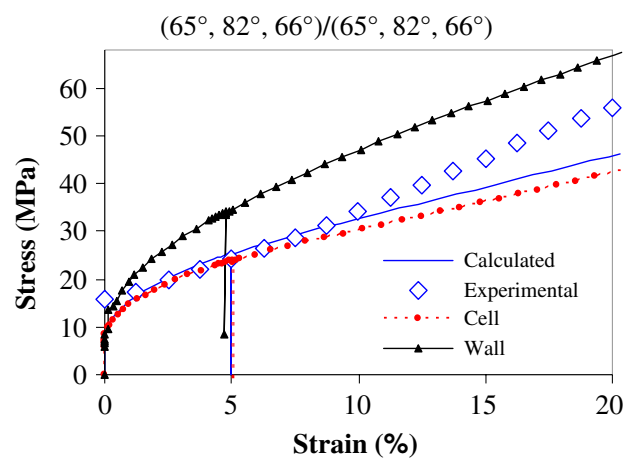

(b)

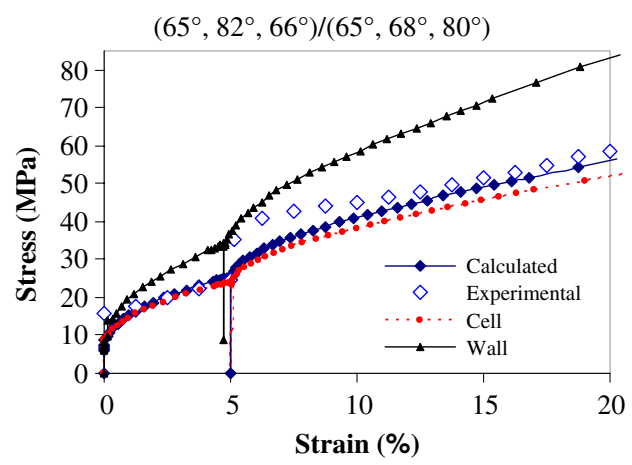

(d) 
Table 3 Material constants for 316L

\begin{tabular}{llllll}
\hline$c_{11}(\mathrm{MPa})$ & $c_{12}(\mathrm{MPa})$ & $c_{44}(\mathrm{MPa})$ & $B(\mathrm{~m})$ & $\rho_{0}\left(\mathrm{~m}^{-2}\right)$ & $y_{c}(\mathrm{~m})$ \\
\hline 245 & 105 & 70 & $5 \times 10^{-10}$ & $1.5 \times 10^{10}$ & $2.8 \times 10^{-9}$ \\
\hline
\end{tabular}

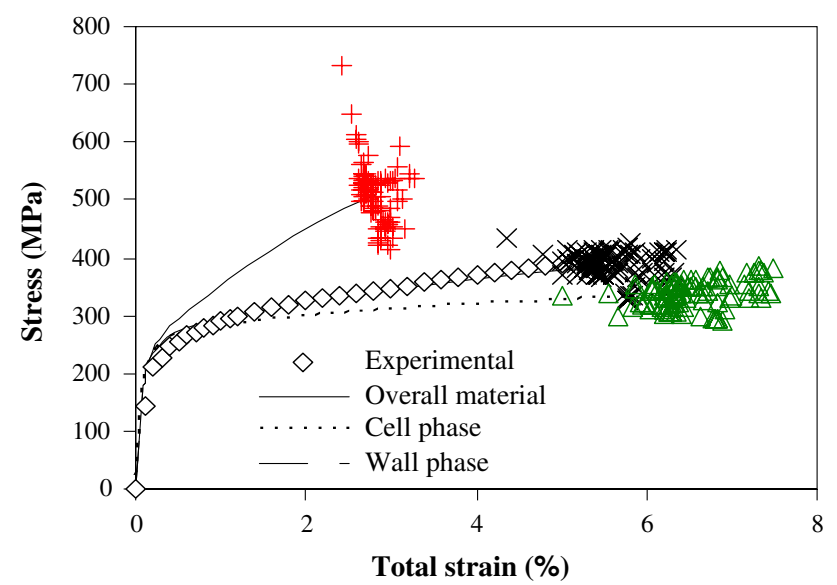

Fig. 5 Experimental and simulated results for tensile test. Scattered points represent the local strains and internal stresses heterogeneity ( $\times: \sigma_{11} / \varepsilon_{11}, \Delta: \sigma_{11}^{c} / \varepsilon_{11}^{c},+: \sigma_{11}^{w} / \varepsilon_{11}^{w}$ ) for macroscopic strains just before elastic unloading

Table 4 Model parameters for single polycrystal

\begin{tabular}{llllllllll}
\hline$a^{g g}$ & $a^{g h}$ & $\tau_{c r 0}(\mathrm{MPa})$ & $k_{L}$ & $f_{0}$ & $f_{\infty}$ & $\gamma^{\mathrm{par}}$ & $\xi$ & $k$ & $\kappa$ \\
\hline 1 & 1.1 & 92 & 0.5 & 0.8 & 0.7 & 2.2 & 0.6 & 25 & 0.5 \\
\hline
\end{tabular}

where $f_{I}$ represents the volume fraction of grain $I$. The material is initially homogeneous. This means that the elastic behaviour and the yield stress are uniform over the polycrystal. The two phases reach their yield limit at the same time which is an assumption that could be discussed. The material becomes heterogeneous since the wall phase is generated. Due to the non-local hardening (through the Eq. 4), high internal stresses are present in the wall phase. The induced microstructure appears with the plastic strain. The contrast between internal stresses within cells and walls appears coherent with the experimental one. Figure 5 shows simultaneously the third-order stresses $\sigma_{11}^{I \alpha}$ for each phase and the resulting mesoscopic stresses at the grain level $\sigma_{11}^{I}$ just before the elastic unloading. This description permits to reveal the strong internal stresses induced in wall phase and the development of heterogeneities during plastic regime. The dislocation density in walls increases more than in cells. The internal stress difference as well as the induced yield stresses are due to this phenomenon. The internal stress inside walls is approximately equal two twice the macroscopic yield stress.

During the first loading, the mean volume fraction in walls [defined by (13)] increases with the plastic deformation due to the development of dislocation density. It varies between

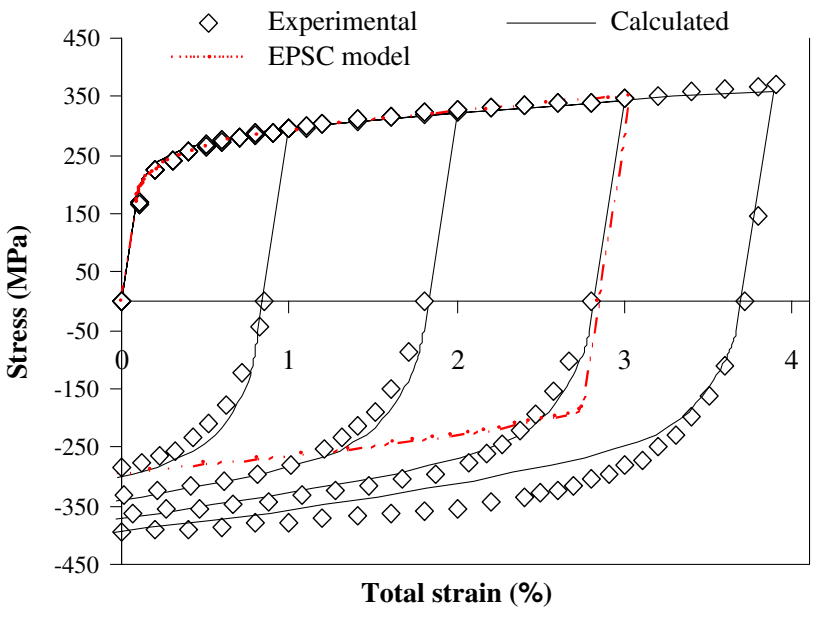

Fig. 6 Comparison between experimental and simulated stress-strain curves

Table 5 Model parameters for single polycrystal

\begin{tabular}{lll}
\hline$H_{1}(\mathrm{MPa})$ & $H_{2}(\mathrm{MPa})$ & $\tau_{c r 0}(\mathrm{MPa})$ \\
\hline 235 & 258 & 85 \\
\hline
\end{tabular}

0.2 at $0 \%$ of plastic deformation to 0.27 after $5 \%$ tensile strain. Linked to the intergranular plastic heterogeneity, the evolution of volume fraction in walls depends on crystallographic orientations at the mesoscopic level. For example, in the less deformed crystallite, the volume fraction increases between 0.2 and 0.28 and, in the most deformed crystallite, the volume fraction increases between 0.2 and 0.25 .

Figure 6 illustrates the simulation results for tensioncompression tests using two different schemes: the EPSC model (for a pre-strain of 3\%) and the present approach. The set of used parameters is summarized in Table 5 for the EPSC model. The hardening matrix contains only two terms $H_{1}$ and $H_{2}$ [15], corresponding to weak and strong interactions between the slip systems. In our calculations, these terms are taken constant during the loading (linear hardening). The material is described by a set of 100 spherical grains with initial random orientation. The EPSC scheme fails to explain and reproduce correctly the softening effects during the compression loading. This method weakly takes into account the formation, the evolution and the stability of induced dislocations microstructures which are strongly path dependent. Even after a moderate strain, i.e. $2 \%$, the disparity between the two approaches is marked. One can observed in Fig. 6 that the present model captures all the essential features (reloading yield stress, macroscopic work hardening) associated with the Bauschinger effect. These comparisons show that the difference can be explained by the internal stresses due to the first uniaxial loading. The Bauschinger effect is stronger with the nonlocal formalism because of 
the high third order stresses which are induced in this case compared to the second-order stresses obtained using the EPSC model. The EPSC does not reproduce quantitatively the Bauschinger effect which is strictly associated with the intragranular stresses. Due to the assumption of homogeneous plastic strain or strain rate inside the grains, the straininduced intragranular microstructures are neglected in the EPSC model. Because of the negative residual stresses due to the first solicitation, the plastic regime begins in cell phase before the wall one. As can be seen, the calculated values are lower than experimental ones but the differences are weak. The residual stresses due to the pre-straining and unloading have a significant effect on the macroscopic stress-strain behaviour through the resolved shear stress. This phenomenon is well captured by the model. The experimental and the predicted macroscopic stresses under reversed loading are always lower than the monotonic stress obtained at the same total strain.

\section{Conclusions}

In this paper, a new approach was developed to predict the elasto-plastic behaviour of single-crystals and polycrystalline aggregates under monotonic and sequential loadings. This approach is based on a two-level transition. The grain is considered as a two-phase material with cell walls (with high density dislocation) and cell interiors (with low dislocation density). A mechanical description of the grain is developed through a micro-meso transition based on a modified elastoplastic self-consistent approach. The intragranular heterogeneities are highlighted by a non-local work-hardening, which is linked to the two-phase description. The new expressions of the hardening law and the local elastoplastic consistent tangent moduli tensor have been determined taking into account internal variables like dislocation densities, volume fraction of cells, etc. Next, a meso-macro transition using a self-consistent model is used to deduce the macroscopic behaviour of the polycristal.

Different numerical results concerning the prediction of macroscopic behaviour of polycrystal and single-crystal with cubic symmetry are calculated. Correct agreement is observed between the simulation data and the experimental results at the meso- and the macroscopic levels. This comparison suggests the relevance of the proposed single-crystal elasto-plastic law and validates the scale transition method used for simple and complex loading paths.

It would be interesting to validate the present model with different two-stage strain path experiments (cross tests, reverse tests). This experimental study is beginning with various cubic alloys. A comparison with prior work of Peeters et al. [23], Viatkina et al. [25], for example, could be interesting to enlighten the relevance of this approach for
FCC polycrystals and validate the scale transition method used. Several improvements of the presented approach are necessary. First, the description using large-scale transformations is required. The evolution of the microstructure should be taken into account in the model. The integration of microstructural morphology into micromechanical approaches via the double-inclusion model proposed by Hori and NematNasser [45] could be a possible (and interesting) solution for next investigations.

\section{References}

1. Aguilar MTP, Cetlin PR, Valle PE, Corrêa ECS, Rezende JLL (1998) Influence of strain path in the mechanical properties of drawn aluminum alloy bars. J Mater Process Technol 80-81: 376-379

2. Yoshida F, Uemori T, Fujiwara K (2002) Elastic-plastic behavior of steel sheets under in-plane cyclic tension-compression at large strain. Int J Plast 18:633-659

3. Feaugas X (1999) On the origin of the tensile flow stress in the stainless steel AISI $316 \mathrm{~L}$ at $300 \mathrm{~K}$ : back stress and effective stress. Acta Mater 47:3617-3632

4. Vincze G, Rauch EF, Gracio SS, Barlat F, Loes AB (2005) A comparison of the mechanical behaviour of an AA1050 and a low carbon steel deformed upon strain reversal. Acta Mater 53:1005-1013

5. Nesterova EV, Bacroix B, Teodosiu C (2001) Microstructure and texture evolution under strain-path changes in low-carbon interstitial-free steel. Metal Mater Trans A 32A:2527-2538

6. Wu PD, MacEwen SR, Lloyd DJ, Jain M, Tugcu P, Neale KW (2005) On pre-straining and the evolution of material anisotropy in sheet metals. Int J Plast 21:723-739

7. Ungar T, Mughrabi H, Rönnpagel D, Wilkens M (1984) X-ray line-broadening study of the dislocation cell structure in deformed [001]-orientated copper single crystals. Acta Metall 32:333-342

8. Ungar T, Groma I (1989) Asymmetric X-ray line broadening of plastically deformed crystals, II. Evaluation procedure and application to [001]-Cu Crystals. J Appl Cryst 22:26-34

9. Hutchinson JW (1970) Elastic-plastic behaviour of polycrystalline metals and composites. Proc Roy Soc Lond A 319:247-272

10. Beradai C, Berveiller M, Lipinski P (1987) Plasticity of metallic polycrystals under complex loading paths. Int J Plast 3:143-162

11. Lipinski P, Berveiller M (1989) Elastoplasticity of micro-inhomogeneous metals at large strains. Int J Plast 5:149-172

12. Van Houtte P (1988) A comprehensive mathematical formulation of an extended Taylor-Bishop-Hill model featuring relaxed constraints, the Renouard-Wintenberger theory and a strain rate sensitivity model. Textures Microstruct 8-9:313-350

13. Gloaguen D, Berchi T, Girard E (2006) Guillén R. Phys Stat Sol (a) 203:R12-R14

14. Zattarin Z, Lipinski P, Rosochowski A (2004) Numerical study of the influence of microstructure on subsequent yield surfaces of polycrystalline materials. Int J Mech Sci 4:377-1398

15. Gloaguen D, François M, Guillén R (2004) Mesoscopic residual stresses of plastic origin in zirconium: interpretation of X-ray diffraction results. J Appl Crystallogr J Appl Cryst 37:934-940

16. Clausen B, Lorentzen T, Leffers T (1998) Self-consistent modelling of the plastic deformation of f.c.c. polycrystals and its implications for diffraction measurements of internal stresses. Acta Mater 46:3087-3098

17. Castelnau O, Francillette H, Bacroix B, Lebensohn RA (2001) Texture dependent plastic behavior of $\mathrm{Zr} 702$ at large strain. J Nucl Mater 297:14-26 
18. Langlois L, Berveiller M (2003) Overall softening and anisotropy related with the formation and evolution of dislocation cell structures. Int J Plast 19:599-624

19. Haddag B, Balan T, Abed-Meraim F (2007) Investigation of advanced strain-path dependent material models for sheet metal forming simulations. Int J Plast 23:951-979

20. Hiwatashi S, Van bael A, Van Houtte P, Teodosiu C (1997) Modelling of plastic anisotropy based on texture and dislocation structure. Comp Mater Sci 9:274-284

21. Mahesh S, Tomé CN, MacCabe RJ, Kaschner GC, Beyerlein IJ, Misra A (2004) Application of a substructure-based hardening model to copper under loading path changes. Metal Mat Trans 35A:3763-3774

22. Mughrabi H (1987) The long-range internal stress field in the dislocation wall structure of persistent slip bands. Phys Stat Sol (a) 104:107-120

23. Peeters B, Seefeldt M, Teodosiu C, Kalidindi SR, Van Houtte P, Aernoudt E (2001) Work-hardening/softening behaviour of b.c.c. polycrystals during changing strain paths: I. An integrated model based on substructure and texture evolution, and its prediction of the stress-strain behaviour of an IF steel during two-stage strain paths. Acta Mater 19:1607-1619

24. Karaman I, Sehitoglu H, Beaudoin AJ, Chumlyakov YI, Maier HJ, Tome CN (2000) Modeling the deformation behavior of Hadfield steel single and polycrystals due to twinning and slip. Acta Mater 18:2031-2047

25. Viatkina EM, Brekelmans WAM, Geers MGD (2008) Numerical analysis of strain path dependency in FCC metals. Comp Mech 41:391-405

26. Muller D, Lemoine X, Berveiller M (1994) Nonlocal behavior of elastoplastic metals: theory and results. J Eng Mat Tech 116: $378-383$

27. Lemoine X, Berveiller M, Muller D (1994) Texture of microstructure in BCG metals for various loading paths. Mat Sci Forum 157-162:1821-1826

28. David F, Aubert I, Lemoine X, Berveiller M (1997) Modelling of elastoplastic polycrystals and aspects of applications. Comp Mat Sci 9:188-198

29. Gloaguen D, François M (2006) Prediction of intragranular strains in metallic polycrystals with two-level homogenisation approach: Influence of dislocations microstructure on the mechanical behaviour. Phys Stat Sol (a) 203:1940-1953

30. Kröner E (1961) On the plastic deformation of polycrystals. Acta Metall 9:155-161
31. Berveiller M, Zaoui A (1979) An extension of the self-consistent scheme to plastically-flowing polycrystals. J Mech Phys Sol 26:325-344

32. Schmitt C, Lipinski P, Berveiller M (1997) Micromechanical modelling of the elastoplastic behavior of polycrystals containing precipitates- Application to hypo- and hyper-eutectoid steels. Int J Plast 13:183-199

33. Hill R (1965) Continuum micro-mechanics of elastoplastic polycrystals. J Mech Phys Solids 13:89-101

34. Nes E (1998) Modelling of work hardening and stress saturation in FCC metals. Prog Mater Sci 41:129-193

35. Essmann U, Mughrabi H (1979) Annihilation of dislocations during tensile and cyclic deformation and limits of dislocation densities. Phil Mag 40:731-756

36. Franciosi $P$ (1985) The concepts of latent hardening and strain hardening in metallic single crystals. Acta Metall 33:1601-1612

37. Ben Zineb T, Arbab Chirani S, Berveiller M (2001) Nouvelle formulation de la plasticité crystalline utilisant une contrainte de reference avec écrouissage. In: Sixteen french conference of mechanic, Nancy, France

38. Lorrain JP, Ben Zineb T, Abd Meriam F, Berveiller M (2005) Ductility loss modelling fo BCC single crystals. Int J Proc 8:135158

39. Gloaguen D, Berchi T, Girard E, Guillén R (2007) Measurement and prediction of residual stresses and crystallographic texture development in rolled Zircaloy-4 plates: X-ray diffraction and the self-consistent model. Acta Mat 55:4369-4379

40. Pollnow D, Penelle R, Lacombe P (1973) Etude des propriétés mécaniques et du taux de consolidation des monocristaux de fer déformés par traction à température ambiante. Lxx 4010:703-714

41. Keh AS, Nakada Y (1967) Plasticity of iron single crystals. Can J Phys 45:1101-1120

42. Choteau M, Quaegebeur P, Degallaix S (2005) Modelling of Bauschinger effect by various constitutive relations derived from thermodynamical formulation. Mech Mat 37:1143-1152

43. Orlans-Joliet B (1989) PhD thesis, Déformation plastique de monocristaux de structure cubique centrée en compression plane, Ecole National des Mines de Paris et de Saint-Etienne

44. Rauch EF (1998) Plastic anisotropy of sheet metals determined by simple shear tests. Mat Sci Eng A241:179-183

45. Hori M, Nemat-Nasser S (1999) On two micromechanics theories for determining micro-macro relations in heterogeneous solids. Mech Mate 31:667-682 